\title{
Total relief of severe left ventricular oufflow obstruction after spontaneous rupture of chordae tendineae in a patient with hypertrophic cardiomyopathy
}

\author{
A Q Araujo, W V Azeredo, E Arteaga, C Mady
}

Heart 2005;91:e35 (http://www.heartinl.com/cgi/content/full/91/5/e35). doi: 10.1136/hrt.2004.057364

In hypertrophic cardiomyopathy (HCM), rupture of mitral chordae tendineae is infrequent and causes acute haemodynamic deterioration. A 38 year old male patient had chordae rupture leading to prolapse of both mitral leaflets and severe regurgitation, without change in symptomatic status. One year before, he had had mild mitral regurgitation and a resting left ventricle outflow tract of $105 \mathrm{~mm} \mathrm{Hg}$ that disappeared in the present evaluation. In this unique case, worsening of mitral regurgitation was counterbalanced by total relief of the severe obstruction. This case report highlights the role of the mitral valve apparatus in the genesis of obstruction in HCM, further stimulating surgical techniques in which mitral repair can be the main procedure.

A male patient aged 38 years had hypertrophic cardiomyopathy (HCM) diagnosed in adolescence. One year before the present evaluation he was mildly symptomatic and taking propranolol $240 \mathrm{mg} /$ day. Doppler echocardiography showed massive hypertrophy (septum, $32 \mathrm{~mm}$; posterior wall, $22 \mathrm{~mm}$ ), a normal left ventricular cavity and fractional systolic shortening, systolic anterior motion of the mitral valve touching the septum, and a resting peak systolic gradient of $105 \mathrm{~mm} \mathrm{Hg}$ in the left ventricular outflow tract (LVOT). Recently, despite unchanged clinical status, the echocardiographic findings became quite different. Both the systolic anterior motion and the gradient (laminar systolic flow in the LVOT) disappeared. New images suggested mitral valve prolapse and rupture of the chordae tendineae. Septal and wall thicknesses decreased to $22 \mathrm{~mm}$ and $14 \mathrm{~mm}$, respectively. The left ventricular diastolic internal diameter increased from $46 \mathrm{~mm}$ to $49 \mathrm{~mm}$; fractional shortening did not change.

Transoesophageal echocardiography confirmed the diagnosis of multiple chordae rupture. Both mitral leaflets were prolapsed leading to a significant regurgitation (fig 1-5).

\section{DISCUSSION}

In HCM, ruptured mitral chordae tendineae is an infrequent complication. Few single case reports can be found in the literature $^{12}$; five cases reported by Zhu et $\mathrm{al}^{3}$ are of note. The rupture can be spontaneous or secondary to infective endocarditis and is generally followed by acute haemodynamic deterioration requiring immediate surgical intervention.

The present case has unique features. Unlike in previously reported cases, the present patient's symptoms did not worsen. The rupture caused prolapse of the medial portions of both mitral leaflets, originating a curious bidirectional regurgitant jet and, importantly, completely relieving the severe LVOT obstruction. We can speculate that the clinical

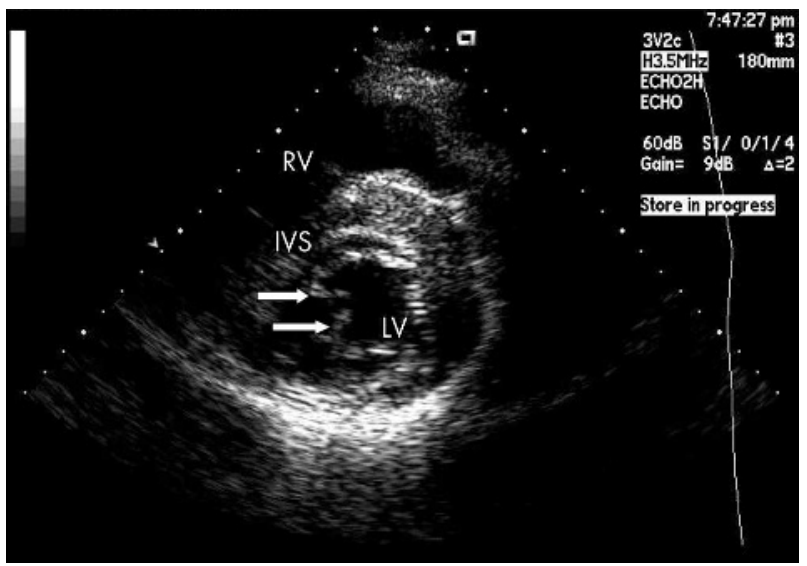

Figure 1 Transthoracic two dimensional parasternal short axis view. The medial portions of the mitral leaflets (arrows) are abnormally positioned during diastole. IVS, interventricular septum; LV, left ventricle; $\mathrm{RV}$, right ventricle.

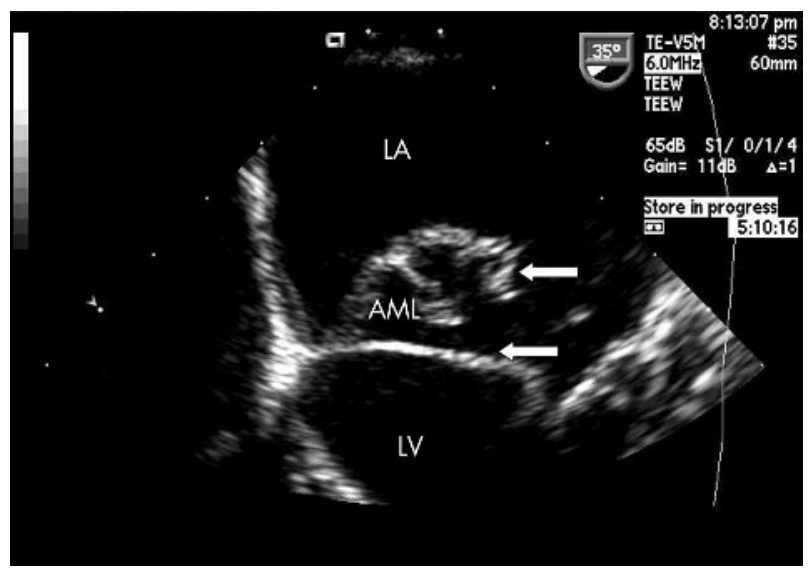

Figure 2 Transoesophageal echocardiogram in systole. In this view, part of the anterior mitral leaflet is normally positioned and part is prolapsed (arrows). AML, anterior mitral leaflet; LA, left atrium.

status was unchanged because the overload imposed by the regurgitation was counterbalanced by the disappearance of the obstructive gradient.

In HCM, the mitral valve apparatus has a critical role in the genesis of LVOT obstruction. A constellation of structural malformations can be present such as enlarged or elongated

Abbreviations: HCM, hypertrophic cardiomyopathy; LVOT, left ventricular outflow tract 


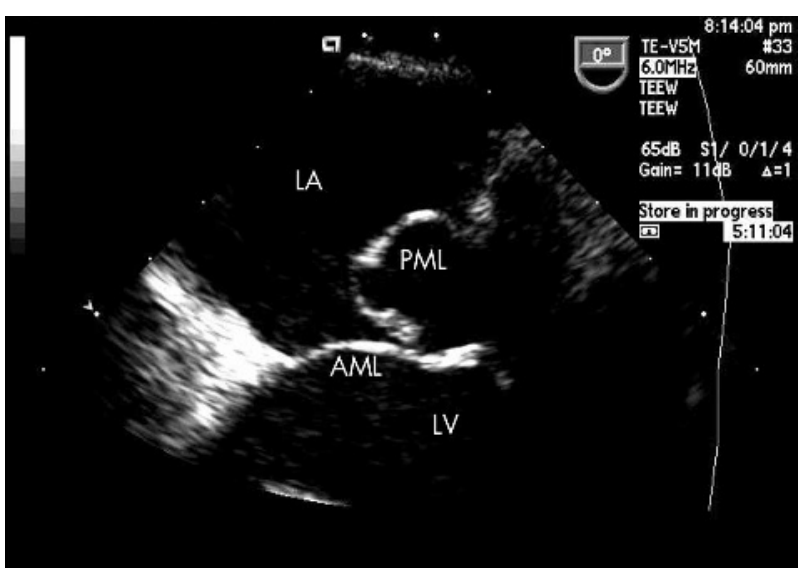

Figure 3 Transoesophageal echocardiogram in systole. In this view, a prolapse of the posterior mitral leaflet is evident. PML, posterior mitral leaflet.



Figure 4 Transoesophageal echocardiogram in systole. In this view, both mitral leaflets appear prolapsed. Image of ruptured chordae is shown (arrow).

leaflets and abnormal papillary muscle insertions. ${ }^{4}$ Coexistence of mitral valve prolapse and HCM is considered infrequent $(3 \%)$. $^{5}$

The best surgical procedure to alleviate obstruction in HCM has been the subject of debate. Septal myectomy (Morrow's surgery), combined myectomy and mitral surgery (repair or replacement), and mitral surgery alone have been the most commonly performed..$^{6-8}$ The choice of the appropriate technique is based on careful preoperative and transoperative morphological evaluation, in addition to the surgeon's preference.

In view of the present case, in which the mitral valve seemed to be the principal and perhaps the only mechanism

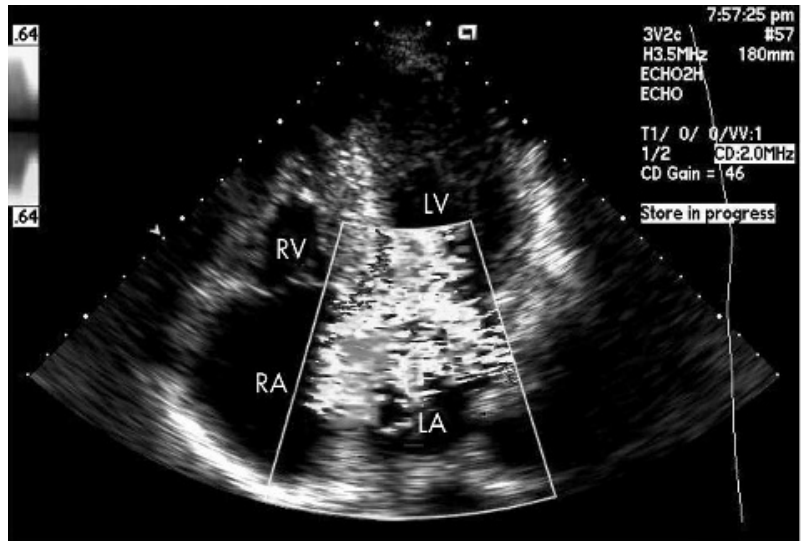

Figure 5 Transthoracic two dimensional apical four chamber view. Colour flow map shows two separate eccentric mitral regurgitation jets.

of obstruction, surgeons must keep in mind the possibility of relieving the obstruction through a single valve repair in selected cases of obstructive HCM.

\section{Authors' affiliations}

A Q Araujo, E Arteaga, C Mady, Heart Institute (Incor), University of Sao Paulo Medical School, Sao Paulo, Brazil

W V Azeredo, Espirito Santo Heart Institute, Brazil

Correspondence to: Dr Aloir Q Araujo, Av Enéas Carvalho Aguiar, 44, 05403-900 Sao Paulo, Brazil; aloirqueiroz@cardiol.br

Accepted 17 December 2004

\section{REFERENCES}

1 Moya JL, Barjau C, Garcia A, et al. Hypertrophic obstructive myocardiopathy complicated by severe mitral insufficiency secondary to rupture of the chordae tendineae. Rev Esp Cardiol 1993;46:840-3.

2 Wakeyama T, Ogawa H, Takaki A, et al. Hypertrophic obstructive cardiomyopathy with ruptured mitral chordae tendineae: a case report. J Cardiol 2004;44:73-9.

3 Zhu WX, Oh JK, Kopecky SL, et al. Mitral regurgitation due to ruptured chordae tendineae in patients with hypertrophic obstructive cardiomyopathy. J Am Coll Cardiol 1992;20:242-7.

4 Klues HG, Maron BJ, Dollar AL, et al. Diversity of structural mitral valve alterations in hypertrophic cardiomyopathy. Circulation 1992;85:1651-60.

5 Petrone RK, Klues HG, Panza JA, et al. Coexistence of mitral valve prolapse in a consecutive group of 528 patients with hypertrophic cardiomyopathy assessed with echocardiography. J Am Coll Cardiol 1992;20:55-61.

6 Cohn LH, Trehan H, Collins JJ, et al. Long-term follow-up of patients undergoing myotomy/myectomy for obstructive hypertrophic cardiomyopathy. Am J Cardiol 1992;70:657-60.

7 Krajcer Z, Leachman RD, Cooley DA, et al. Septal myotomy-myectomy versus mitral valve replacement in hypertrophic cardiomyopathy: ten-year follow-up in 185 patients. Circulation 1989;80:157-64

8 Stassano P, Di Tommaso L, Triggiani D, et al. Mitral valve replacement and limited myectomy for hypertrophic obstructive cardiomyopathy: a 25-year follow-up. Tex Heart Inst J 2004;31:137-42. 